\title{
Probability of mixing up the nearest neighbor robot under target enclosure by robot swarm
}

\author{
Masao Kubo \\ Computer Science, National Defense Academy of Japan, Yokosuka, Kanagawa, Japan \\ Hiroshi Sato \\ Computer Science, National Defense Academy of Japan, Yokosuka, Kanagawa, Japan \\ Akihiro Yamaguchi \\ Department of Information and Systems Engineering, Fukuoka Institute of Technology Fukuoka, Japan \\ Eugene Kitamura \\ Computer Science, National Defense Academy of Japan, Yokosuka, Kanagawa, Japan \\ Akira Namatame \\ Computer Science, National Defense Academy of Japan, Yokosuka, Kanagawa, Japan \\ E-mail: \{masaok,hsato,ed12005, nama\}@nda.ac.jp, aki@fit.ac.jp
}

\begin{abstract}
This paper analyzes conditions in which a robotic swarm with a simplest communication device can succeed to enclose a target. The robots have to know directions to their each nearest neighbors. Each robot has a same signal emitter like a torch and they can observe the sum of the intensity of their torches. We analyze the probability to the mistake of the direction when a robot adopts the direction of the strongest intensity of the signal as the direction of the nearest neighbor because we expect this approach makes the robot swarm more simple and scalable. The computer simulations show the case of the success.
\end{abstract}

Keywords: target enclosure, swarm robotics, low cost robot design, collective intelligence

\section{Introduction}

This paper analyzes conditions in which a robotic swarm with a simplest communication device can succeed to enclose a target. The swarm is equipped with a same limited transmission range signal emitter. This communication system could make a large robot swam cheaper and scalable. On the other hand, it suffers a lower performance because they 
could not observe any correct information. In this paper, this low cost robot design concept is applied to a robot swarm to enclose a target.

The target enclosure task is useful for monitoring of disaster sites. We focused on the study of Takayama et al. ${ }^{3}$. In their model, each robot needs information of directions to only one neighbor and to its target. Recently, a research used a hybrid system theory and showed that robots controlled by this method which references the nearest neighbor can also enclose a target ${ }^{1}$.

In making a cheap and scalable component for a robot swarm a limited range homogeneous signal emitter is focused. We call it the torch system. For example, Kilobot ${ }^{2}$ has the same small LED emitter as a communication device and a flock of 1000 Kilobots can form a large shape. However, the transmission range of their LED light is about 6 robot length. Swarmbot ${ }^{4}$ proposes that the robot having a homogeneous light can collect the sufficient number of robots to solve a task. Each robot has the same color lights around its body. The intensity of light becomes strong as the number of robots increase. If the intensity of light is too strong a robot will not join the group.
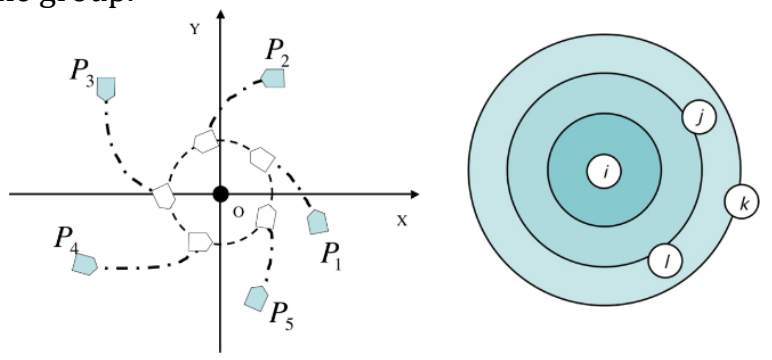

Fig.1 Model of Takyama's Fig.2 Diffusion of algorithm: $\alpha, \beta$. light of torch

The advantage of this torch system is its high procurability. The very limited transmission range provides less interference of communication. Therefore, emitters which use the same signal band can be installed into many robots. Also, its small energy consumption is an advantage. The smaller the communication range, the smaller the energy consumption.
In this paper, we examine a robotic swarm with torch system for the target enclosing task. We adopt Takayama's work for the control scheme of robot but the referencing robot is different in this work. We propose the most powerful signal direction to be the direction of the referencing robot. We call this the "MOPS" direction. In this paper, we show that the proposed robotic swarm can enclose a target successfully by computer simulation. We discuss the probability of mixing up the nearest neighbor robot under the target enclosure task by robot swarm.

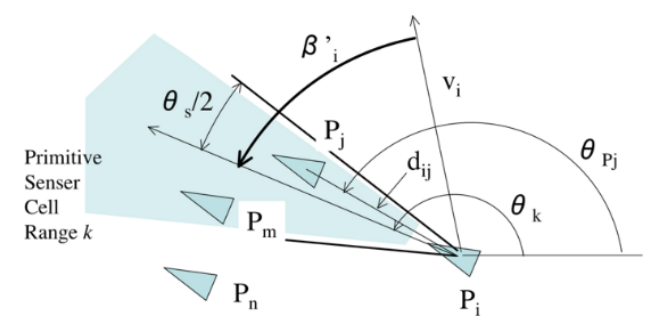

Fig.3 The most powerful signal (MOPS) direction

\section{Takayama's target enclosing model}

Firstly, Takayama's target enclosing model is explained. On a two-dimensional plane, let there be only one target $O$ at the origin and $n$ robots. Robots are numbered counterclockwise as $P_{1, . .,} P_{n}$, and $r_{i}$ is the position vector of the robot $P_{i}$.

To achieve this task, Takayama et al. ${ }^{3}$ proposed the following model. Each robot determines its control input, speed $v_{i}$, and angular velocity $\omega_{i}$ using two aspects of angular information: relative angles with respect to the target and an anterior neighboring robot, denoted as $\alpha_{i}$ and $\beta_{i}$, respectively. As a result, rotational movement occurs with a central focus on the target.

$$
\begin{aligned}
& v_{i}=f \beta_{i} \\
& \omega_{i}=\frac{v_{i}}{\bar{r}}-k \cos \left(\alpha_{i}\right)
\end{aligned}
$$

where the parameters $\bar{r}, k$, and $f>0$ as specified beforehand. $P_{i+1}$ is the robot to which $P_{i}$ refers, and $\bar{r}$ 
is the expected distance to the target. In Takayama et al.'s model, the $i$-th robot refers to the $i+1$-th robot, and the $n$-th robot refers to the first robot $P_{1}$.

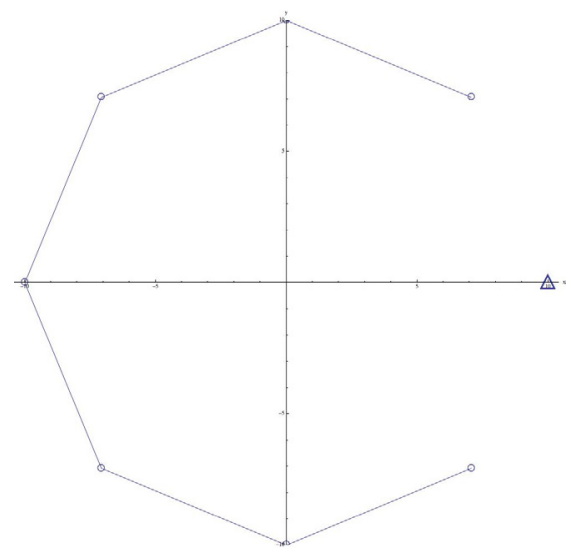

Fig.4 The location of agents when they enclose the target completely $(|N|=8) . \triangle$ indicates the reference agent and $\bigcirc$ means its neighbor agents.

\subsection{Nearest neighboring robot as the reference}

Ref.1 examined a different reference robot scheme in which each robot considers its nearest neighboring robot as its reference robot. Each robot controls itself as described in equation 1 and 2, but it chooses its nearest neighbor as its reference robot. This system has higher scalability because the individual robots need not be identified to observe the nearest robot.

\section{The proposed reference model: the most powerful signal direction model, MOPS model}

We propose an alternative robotic swarm for the target enclosure task. Each robot carries the torch with the same specification. Additionally we propose a most powerful direction of their signal is used as the direction of the referencing robot. First, we explain the torch which the robots have.

\section{1. Torch}

Every agent has a same torch and it can turn on/off its torch. The maximum intensity of all the torch is same and known beforehand. We suppose that the speed of propagation of the signal of the torch is so fast that an agent can observe the signal immediately. As shown in Fig. 2 during the flight of the signal, the power of the signal is attenuated. The signal propagation function $p(d)$ is described as follows.

$$
p(d)=\left\{\begin{array}{cc}
\gamma^{(d / L)} /(d / L)^{2} & d>L \\
1 & \text { otherwise }
\end{array}\right.
$$

where $d$ is the distance from the emitter, $L$ is the length of an agent, $\gamma$ is the attenuation coefficient per $L$. The equation 3 represents the spherical diffusion of a light. An agent closer to an emitter receives a stronger signal. Target enclosing model with MOPS direction reference model

We explain the procedure of receiving a signal in the proposed method.

Agent $P_{i}$ uses the most powerful signal direction instead of the angle from $P_{i}$ to $P_{j}\left(=\beta_{i}\right)$. Also, we call the angle $\beta_{i}^{\prime}$. Fig. 3 shows this angle. Now, we suppose agent $P_{i}$ has several signal receivers. We call it sensor primitive. The direction of $k$-th sensor primitive $s p_{k}$ is $\theta_{k}$. If $\left|\theta_{k^{-}} \theta_{i j}\right| \leqq \theta_{s} / 2$, the sensor primitive $s p_{k}$ can sense the signal of $P_{j}$. We call $\theta_{s}$ a visible range of sensor primitive. The power of sensor primitive $s p_{k}, s_{k}$ is

$$
S_{k}=\sum p\left(d_{i, m}\right)
$$

where $d_{i, m}$ is the distance between $P_{m}$ and $P_{i}$ and $p$ is the signal propagation function in eq.3. $\beta_{i}^{\prime}$ is the substitution of $\beta_{i}$.

$$
\beta_{i}^{\prime}=\angle v_{i} P_{i} \theta_{\arg \max _{k} s_{k}}
$$

\section{Analysis of the proposed direction reference model}

In this section, we describe the result of analysis of the proposed MOPS reference model. In this section, we clarify that it is possible for a set of adequate sensor primitives to detect the nearest neighbor agent 
if the locational variance of the neighbor robots is small.

\section{1. Definition of this target enclosing task}

The following 2 conditions are defined for a successful target enclosing. For any agent $P_{i}$, the first condition is that the distance between $P_{i}$ and the target at $O$ is equal to $\bar{r}$. The second condition is that for any agent $P i$ the $\angle P_{i} O P_{j}=2 \pi /|N|$ where $P_{j}$ is the nearest neighbor agent of $P_{i}$.

Now, we suppose that an agent, $i=0$ is at $\left(r_{i}, \theta_{i}\right)=$ $(\bar{r}, 0)$. We call the agent with $i=0$ the reference agent. Also other agents are called the neighbor agents. When they enclose the target successfully, the locations of the neighbor agents are $\left(r_{i}, \theta_{i}\right)=\left(\bar{r}, i C_{\theta}\right)$, $C_{\theta}=2 \pi /|N|, \quad i=1, \ldots,|N|-1$. We call these locations regular locations. Even if the reference agent moves while the other agents enclose the target, the relative position of the reference agent to the other agents remain the same. The distance to a neighbor agent $\mathrm{i}, \mathrm{i}$ $=1, \ldots,|\mathrm{N}|-1$ is

$d_{i}=\sqrt{2 \bar{r}^{2}\left(1-\cos \left(i C_{\theta}\right)\right)}$.

Therefore,

$d_{i=1}=d_{i=|N|-1}<d_{i \neq 0,1, N \mid-1}$

We think of 2 nearest neighbor agents for the reference agent when they enclose the target.

\section{2. Fluctuation of signal power caused by displacement of neighbor agents}

The signal power of neighbor agent $i$ at the reference agent can be approximated by Taylor expansion. It is described as follows.

$S_{i}=A_{i}+K_{i} \Delta_{i}, A_{i}=\gamma^{d_{i} / L} L^{2} / d^{2}{ }_{i}, K_{i}=\left[k_{i, r}, k_{i, \theta}\right]$

$k_{i, r}=-\left(\frac{2 L \gamma^{\frac{d_{i}}{L}} \bar{r}}{d_{i}^{5}}\right)\left(1-\cos \left(i C_{\theta}\right)\right)\left(\bar{r}^{2} \log (\gamma)\left(\cos \left(i C_{\theta}\right)-1\right)+L d_{i}\right)$

$k_{i, \theta}=-\left(\frac{2 L \gamma^{\frac{d_{i}}{L}} \bar{r}^{2}}{d_{i}^{5}}\right) \sin \left(i C_{\theta}\right)\left(\bar{r}^{2} \log (\gamma)\left(\cos \left(i C_{\theta}\right)-1\right)+L d_{i}\right)$

where $\Delta_{i}=\left[\Delta r_{i}, \Delta \theta_{i}\right]^{T} . \Delta r_{i}$ is a displacement along the target direction from its regular position, $\Delta \theta_{i}$ is a circumferential displacement from its regular position. We assume that the displacements $\Delta r_{i}, \Delta \theta_{i}$ follow the normal distributions having average 0 and variance $\sigma_{r_{i}}^{2}, \sigma_{\theta_{i}}^{2}$ respectively. Also, we assume that the fluctuation of signal power at the reference agent follows a normal distribution having $\sigma_{i}$. In this case, the average of power of the signal at the reference agent is $A_{i}$, and its distribution $\sigma^{2}{ }_{i}$. is

$\sigma^{2}{ }_{i}=K_{i} \Sigma_{i} K_{i}^{T}=k_{i, r}^{2} \sigma_{r_{i}}^{2}+k_{i, \theta}^{2}+\sigma_{\theta_{i}}^{2}$

where $\Sigma_{i}=\operatorname{diag}\left(\sigma_{r_{i}}^{2}, \sigma_{\theta_{i}}^{2}\right)$

The deviation of the signal at the reference agent can be calculated if $|N|$ and $\sigma_{r_{i}}, \sigma_{\theta_{i}}$ are known. We suppose that $I_{\mathrm{s}}$ means the set of agents which are in visible range of sensor primitive $s$. The average $\bar{p}_{s}$ and the standard deviation $M_{s}$ of signal at the reference agent are described as follows.

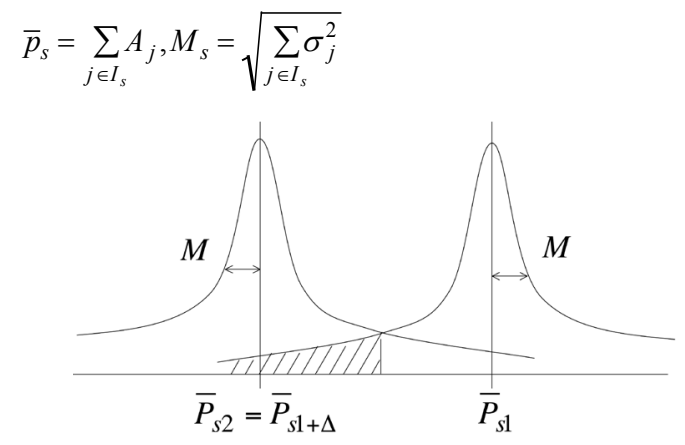

Fig.5 The probability with which a farther neighbor is recognized as a closer neighbor.

\section{3. Limit of variance of signal under which an agent can detect the nearest neighbor agents}

We assume that there are 2 sensor primitives $\mathrm{s} 1$, s2 of the reference agent. Each sensor primitive observes one of the nearest neighbor agents in equation 7 . Also visible ranges of s1 and s2 are so narrow that it receives signal from only one of the nearest neighbor agents, namely, $\left|I_{\mathrm{s} 1}\right|=\left|I_{\mathrm{s} 2}\right|=1$.

Now, we suppose that a small displacement at the nearest neighbor agents occurs while all of the agents move. We assume that the difference of distance between the 2 nearest neighbor agents is $\Delta$. 
In this case, the condition for the reference agent to choose a sensor primitive correctly for observing the closer agent can be deduced by using the $3-\sigma$ rule.

$\bar{p}_{s 1}-k M>\frac{\bar{p}_{s 1}+\bar{p}_{s 2}}{2}$

where $\bar{p}_{s 1} \geq \bar{p}_{s 2}$ and $k$ is a positive constant. Here $k=3$ for the rest of this paper.

Therefore, if the standard deviation of the sensor primitive $M$ is smaller than $M_{\text {Limit }}$, the reference agent can almost certainly select the correct sensor primitive. $M_{\text {Limit }}$ is described as follows.

$M \leq M_{\text {Limit }}=\frac{p\left(d_{1}\right)-p\left(d_{1}+\Delta\right)}{2 k}$

$M_{\text {Limit }}$ is changed by signal's attenuation coefficient $\gamma$. Fig.6 illustrates $M$ and $M_{\text {Limit }}$ while $\gamma$ changes. $M$ with $\sigma_{\theta_{i}}=0.5,1$ and 2 degree are illustrated by lines with markers,$+ \bigcirc$, and $\square$ respectively $.|N|=8, \bar{r}$ $=10 \mathrm{~m} . \sigma_{r_{i}}=0.1 \mathrm{~m}$.

The variance of signal power at the reference agent becomes smaller as the $\sigma_{\theta_{i}}$ becomes small.

The solid and dashed lines represent $M_{\text {Limit }}$ with $\Delta=1 \mathrm{~m}$ and $2 \mathrm{~m}$ respectively. In order to detect the difference in distance of $\Delta \mathrm{m}$ between the nearest agents with high certainty, the variation of the received signal $M$ must be smaller than $M_{\text {Limit }}$ lines. For example, Fig.6 shows that even if the nearest neighbor agent's location is distributed $\sigma_{\theta_{i}}=0.5$ degrees, the reference agent can detect $1 \mathrm{~m}$ difference between the 2 nearest agents for any $\gamma$. On the other hand, the reference agent cannot detect such difference if the nearest neighbor agent' $s$ location is distributed $\sigma_{\theta_{i}}=1$ and 2 degrees.

By this experiment, we expect that if the $\sigma_{\theta_{i}}$ is sufficiently small, the reference agent that is $\Delta$ closer than the 2nd nearest neighbor agent can be selected by the sensor primitive with the strongest intensity signal.

\section{Conclusions}

In this paper, we examine a robotic swarm with the torch system, which is a simple communication device, for the target enclosing task. The direction of the nearest neighbor is an important information for this task. We can expect that if the fluctuation of an agent is sufficiently small and the gap of distance between the nearest and the second nearest neighbor agent $(\Delta)$ is given, the agent can select the correct sensor primitive which observes the nearest neighbor agent.

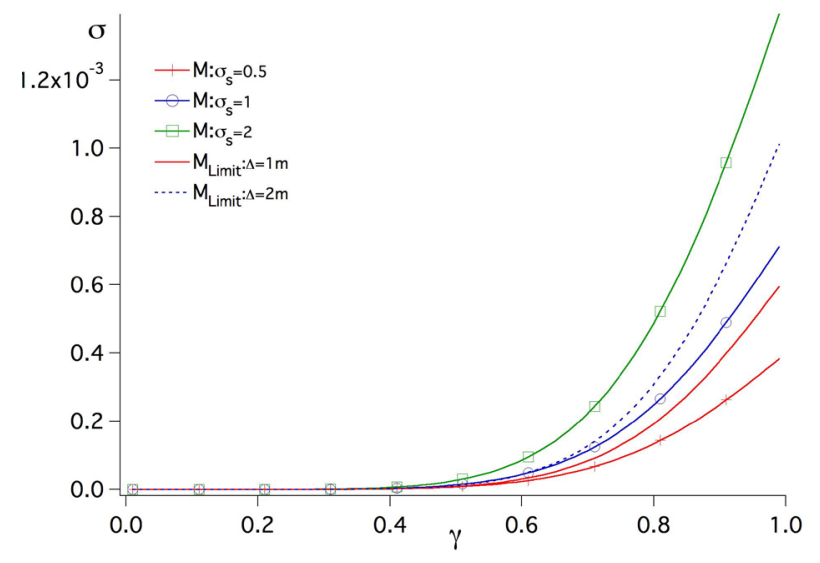

Fig.6 Limit of variance of signal under which an agent can detect the nearest neighbor agents

\section{References}

1. M. Kubo, H. Sato, T.Yoshimura, A.Yamaguchi, T. Tanaka "Multiple targets enclosure by robotic swarm", Robotics and Autonomous Systems 62,12941304(2014)

2. M.Rubenstein, C.Ahler, N.Hoff, A.Cabrera, R.Nagpal, "Kilobot: A low cost robot with scalable operations designed for collective behaviors", Robotics and Autonomous Systems, 62, 996-975,(2014).

3. Y. Takayama, S. Yamamoto, T. Takimoto, "Distributed Formation Control of a Nonholonomic Multiagent System for Target-enclosing Operations", Proceedings of 9th SICE System Integration Division Annual Conference, SY0015, 679-680(2008)

4. F. Ducatelle, A. Förster, G. D. Caro, and L. Gambardella, "New task allocation methods for robotic swarms", 9th IEEE/RAS Conference on Autonomous Robot Systems and Competitions,(2009). 